FROM GIGABYTES TO BYTES: AUTOMATED DENOISING AND FEATURE IDENTIFICATION IN ELECTRON TOMOGRAMS OF INTACT BACTERIAL CELLS

By

$$
\begin{aligned}
& \text { Rajesh Narasimha, Iman Aganj } \\
& \text { Mario Borgnia, Guillermo Sapiro }
\end{aligned}
$$

Steven McLaughlin, Jacqueline Milne

and

Sriram Subramaniam

IMA Preprint Series \# 2145

(November 2006)

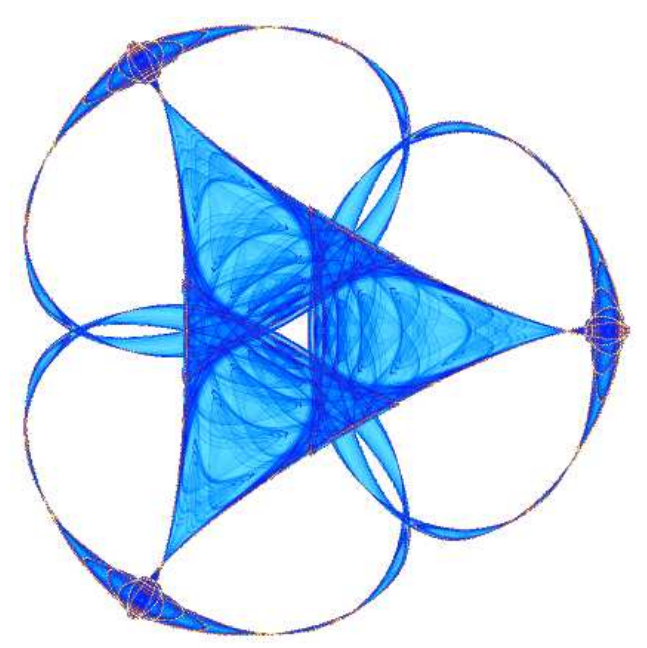

INSTITUTE FOR MATHEMATICS AND ITS APPLICATIONS

UNIVERSITY OF MINNESOTA 400 Lind Hall

207 Church Street S.E.

Minneapolis, Minnesota 55455-0436

Phone: 612-624-6066 Fax: 612-626-7370

URL: http://www.ima.umn.edu 


\title{
FROM GIGABYTES TO BYTES: AUTOMATED DENOISING AND FEATURE IDENTIFICATION IN ELECTRON TOMOGRAMS OF INTACT BACTERIAL CELLS ${ }^{1}$
}

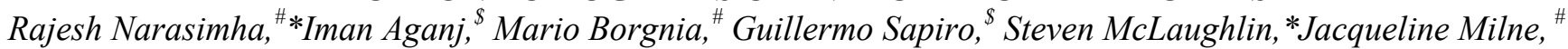 \\ and Sriram Subramaniam ${ }^{\#}$ \\ ${ }^{\#}$ Laboratory of Cell Biology, Center for Cancer Research, National Cancer Institute, Bethesda, MD, \\ ${ }^{*}$ Georgia Institute of Technology, Atlanta GA ${ }^{\$}{ }^{\$}$ University of Minnesota, Minneapolis, $M N$ \\ \{rajesh,swm\}@ece.gatech.edu, \{iman,.guille\}@umn.edu,borgnia@mail.nih.gov,\{jmilne,ss1\}@nih.gov
}

Abstract: Advances in automated data acquisition in electron tomography have led to an explosion in the amount of data that can be obtained about the spatial architecture of a variety of biologically and medically relevant objects with resolutions in the "nano" range of 10-1000 $\mathrm{nm}$. The development of methods to automatically analyze the vast amounts of information contained in these tomograms is a major challenge since the electron tomograms are intrinsically very noisy. A fundamental step in the automatic analysis of large amounts of data for statistical inference is to segment relevant $3 \mathrm{D}$ features in cellular tomograms. Procedures for segmentation must work robustly and rapidly in spite of the low signal to noise ratios inherent to biological electron microscopy. This work first evaluates various non-linear denoising techniques on tomograms recorded at cryogenic temperatures. Using datasets of bacterial tomograms as an example, we demonstrate that non-linear diffusion techniques significantly improve the fidelity of automated feature extraction. Our approach represents an important step in automating the efficient extraction of useful information from large datasets in biological tomography, and facilitates the overall goal of speeding up the process of reducing gigabyte-sized tomograms to relevant byte-sized data.

Keywords: Electron tomography, denoising, feature extraction, diffusion, automated techniques, template matching.

\section{INTRODUCTION}

Electron tomography (ET) is an emerging tool to describe the three-dimensional (3D) architectures of large molecular complexes, viruses and cells. ET is a general method for 3D reconstruction of electron transparent objects from a series of projection images recorded with a transmission electron microscope. For most biological applications electron tomograms are obtained at relatively poor signal to noise ratios [1].

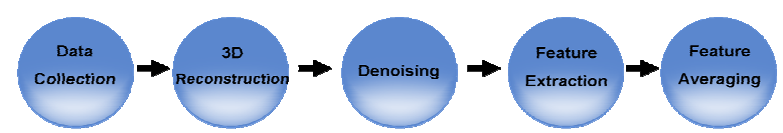

Fig. 1. Imaging Pipeline.

The typical steps in imaging and image processing are highlighted in Fig. 1. Data collection normally involves recording a series of images at different relative tilts of beam and specimen. The electron microscope provides $2 \mathrm{D}$ projection images. Tomograms are generated by computational analysis of a series of electron micrographs taken at varying tilt angles, usually from $-70^{\circ}$ to $+70^{\circ}$. Using algorithms that implement weighted back projection (WBP) or Simultaneous Iterative Reconstruction Technique (SIRT), one can obtain $3 \mathrm{D}$ volumes of the specimen from projection images.

As techniques for data collection and tomogram reconstruction are becoming more streamlined, the development of automatic and semi-automatic methods to analyze the enormous amounts of information in these tomograms remains a major challenge [1]. Efforts to establish tools for quantitative interpretation of tomograms are beginning to be applied to a range of biological problems, as reviewed recently [2]. A long-term goal of this type of electron tomography is to interpret the spatial arrangement of the constituent molecular and cellular components at the highest resolution possible. A fundamental step in the automatic analysis of large amounts of data for statistical inference is to employ robust methods for $3 \mathrm{D}$ segmentation that can work well at low SNR. The target of these strategies is to improve the signal as much as possible relative to the noise level to mine 3D information from complex tomograms.

This work investigates image/transform-domain denoising techniques and feature extraction in the context of electron tomography of whole bacterial cells. Previous studies have already demonstrated the value of image denoising in various $2 \mathrm{D}$ and $3 \mathrm{D}$ datasets [3-7]. Our goal here is to first evaluate the performance of a range of denoising techniques on electron tomograms recorded at cryogenic temperature, and then use the denoised data in the segmentation algorithm. For analysis and feature extraction, we have used tomograms of Bdellovibrio bacteriovorus, a small bacterium, recorded at cryogenic temperatures, and reconstructed using WBP-based approaches. We report here on the relative merits of a variety of denoising algorithms on the detection, clustering, and computation of the spatial distribution of ribosomal complexes in the bacterium, and have compared their performance using single-image SNR estimation and Fourier ring correlation techniques. The performance of these denoising methods was tested on two sets of tomograms that were recorded at similar electron optical conditions. We found that the 3D nonlinear anisotropic diffusion (NAD) algorithm (described in Section 3) significantly improved the SNR while retaining the overall architectural information in the tomograms. Following the denoising, automated segmentation is achieved using a template matching based feature extraction method which considers intensity and mean-squared error between the template and the ribosome in the tomogram. We validate our approach by comparison of semi-automated and automated segmentation procedures on these two sets of tomograms. Furthermore, we present quantitative analysis such as ribosomal distribution in $3 \mathrm{D}$ which provides insights into the spatial architecture of intact cells.

\section{DATA COLLECTION AND RECONSTRUCTION TECHNIQUES}

We imaged Bdellovibrio cells preserved by embedding in a thin layer of vitrified ice. For specimen preparation, an aliquot of cell culture $(3 \mu \mathrm{l})$ was laid on a thin layer of holey carbon supported on a $3 \mathrm{~mm}$ copper grid (Quantifoil MultiA, Micro Tools $\mathrm{GmbH}$, Germany). Excess liquid was blotted off with filter paper after $1 \mathrm{~min}$ incubation and the grid was plunge frozen in liquid ethane cooled by liquid nitrogen. Specimens were maintained and imaged at liquid nitrogen temperatures in a Polara microscope

\footnotetext{
${ }^{1}$ Work partially supported by NSF and NIH
} 
(FEI Corp., OR, U.S.A.) equipped with a field emission gun operating at $300 \mathrm{kV}$. Series of low dose $\left(1-2 \mathrm{e}^{-} / \mathrm{A}^{2}\right)$ projection images of the frozen specimen, tilted over an angular range of $\pm 69^{\circ}$ at fixed $3^{\circ}$ intervals, were recorded in a $2 \mathrm{~K}$ by $2 \mathrm{~K}$ CCD camera located at the end of a GIF 2000 (Gatan Inc., Pleasanton, CA, U.S.A.) energy filtering system. The effective magnification was $22500 \mathrm{X}$, equivalent to a pixel size of $0.63 \mathrm{~nm}$ at the specimen level, and the applied under-focus was $15 \mu \mathrm{m}$. Full resolution images were aligned with the aid of colloidal gold fiducial markers deposited on the carbon prior to specimen preparation. Three dimensional reconstructions were done by weighted back projection of aligned images binned by $4 \times 4$. The package IMOD [8] was used for alignment and reconstruction.

\section{DENOISING AND SEGMENTATION METHODS}

We now provide a brief explanation of the denoising techniques used to analyse the tomographic data. Our main goal here is to apply existing denoising techniques to $3 \mathrm{D}$ tomograms recorded at cryogenic temperature. This is particularly of great interest since one denoising algorithm will not perform uniformly well on diverse datasets that have been collected using a variety of specimens and acquisition conditions. The putative ribosomes were readily distinguished from the other macromolecular complexes since they are more electron dense. The rest of the cytoplasm is just as packed with other complexes, possibly of similar size to ribosomes, but they have less contrast. The tomograms also need to be qualitatively examined by a biological expert to determine whether the features of interest are well preserved after denoising. Thus we used both qualitative and quantitative measures to choose the optimal denoising algorithm. We first implemented and compared the performance of non-linear anisotropic diffusion [3] and complex diffusion algorithms [4] on electron tomograms. It has been demonstrated in [9] that nonlinear diffusion methods significantly improve the SNR. The equations for anisotropic diffusion in $3 \mathrm{D}$ are given as $I_{t}=\nabla \cdot(c(x, y, z, t) \nabla I)$, where and subscripts denote derivatives,

$$
c(x, y, z, t)=1 /\left(1+\frac{|\nabla I|^{2}}{\kappa^{2}}\right) \text { or } c(x, y, z, t)=\mathrm{e}^{-\frac{|\nabla I|^{2}}{2 \kappa^{2}}}
$$

$\kappa$ is a conductance parameter and $I=I(x, y, z, t)$ is the $3 \mathrm{D}$ tomogram. For the complex diffusion,

$$
I_{t}=\nabla \cdot(c(\operatorname{Im}(I)) \nabla I) \text { with } c(\operatorname{Im}(I))=e^{i \theta} /\left(1+(\operatorname{Im}(I) / k \theta)^{2}\right)
$$

where $\operatorname{Im}(I)$ is the imaginary value of $I, \theta$ is the phase angle and $k$ is the threshold parameter. The noisy tomogram is used as initial condition for the above non-linear partial differential equations. In another tested method of denoising, the phase data is preserved [5] by applying the continuous wavelet transform and by using log Gabor functions to construct symmetric/anti-symmetric wavelet filters. The process determines a noise threshold at each scale, and by shrinking the magnitudes of the filter response vectors, phase information can be preserved after reconstruction. The other denoising methods we used for comparison are based on translation invariant wavelets with soft thresholding [6] and Bayes least-squares Gaussian scale mixtures (BLS-GSM) [7] where we used steerable pyramid wavelets and Wiener filtering. We used Wavelab and BLS-GSM MATLAB software publicly available for both these methods with modifications. For all these methods we obtained the highest SNR by varying the parameters over a valid range and also making sure that the spatial features of interest are well retained from the biological point of view. We considered two sets of Bdellovibrio tomograms reconstructed using WBP.
The effect of denoising is evident by comparing Fig. 2(c) vs. 2(d) and Fig. 3(e) vs. 3(f) where we can observe greater definition of intracellular components in the denoised tomographic slices. The quantitative analysis procedures such as peak-SNR (PSNR) and $\sqrt{M S E}$ assume that a clean reference image is available. For quantitative analysis of the denoising methods we implement single-image SNR estimation, [10], since we are working with tomograms which are essentially stacks of $2 \mathrm{D}$ slices of the reconstructed volume. We compute the SNR of each slice using various denoising techniques and the result shown in Fig. 2(a) is an average SNR over 10 consecutive slices. The single-image SNR estimation method assumes that the noise is white and uncorrelated from pixel to pixel. The SNR is given as [10]

$$
S N R=\frac{\rho_{12}}{\left(1-\rho_{12}\right)}=\frac{\phi_{11}^{N F}(0,0)-\mu_{1}^{2}}{\sigma_{1}^{2}-\left(\phi_{11}^{N F}(0,0)-\mu_{1}^{2}\right)}=\frac{\phi_{11}^{N F}(0,0)-\mu_{1}^{2}}{\phi_{11}(0,0)-\phi_{11}^{N F}(0,0)}
$$

where $\phi_{11}(0,0)$ and $\phi_{11}^{N F}(0,0)$ are the autocorrelation function (ACF) of the noisy image and the noise free (NF) image respectively. $\mu_{1}$ and $\sigma_{1}$ are the mean and variance of the noisy image. Since we do not have a noise-free reference image, $\phi_{11}^{N F}(0,0)$ is estimated by assuming that its value is same as the $\mathrm{ACF}$ of the neighboring offsets, i.e., $\left\langle\phi_{11}^{N F}(0,0)\right\rangle \approx\left(\phi_{11}(1,0)+\phi_{11}(0,1)\right) / 2$. This estimate is reasonable if the

ACF changes slowly at the origin which is the case for cryogenic tomograms. Single image SNR estimation on a cryo tomogram in Fig. 2(a) suggests that the nonlinear anisotropic diffusion algorithm (PM1and PM2 [3]) performs the best in terms of the SNR. For e.g., the SNR of the noisy tomograms were improved significantly from $1.2 \mathrm{~dB}$ as shown in the figure. Furthermore, we also compute the Fourier ring correlation (FRC) [11] to compare various reconstruction and denoising methods as in Fig. 2(b). It can be observed that nonlinear anisotropic diffusion exhibits good performance at low frequencies. It should be noted that we are dealing with noisy tomograms and hence FRC may not be an accurate similarity measure at high frequencies.

Next we compare both semi-automated and automated feature extraction methods since our objective is to develop automatic procedures from data collection to feature extraction that can be integrated into the imaging pipeline. In testing the value of denoising, we used semi-automated and automated detection of putative ribosome as a measure of performance.

Semi-automated segmentation procedure: Tomograms were segmented using the Amira Visualization software [12] which executes a simple procedure of $3 \mathrm{D}$ region-growing from user-marked voxels and with user-defined range. The userdesignated regions of the tomogram were also subjected to thresholding. To obtain the coordinates, for each ribosome, the $\mathrm{z}$ range of the volume had to be counted and the "middle slice" was used to obtain the $(x, y, z)$ coordinates. For an even $z$-range, the center was estimated from the two middle slides, using averaging. The semi-automated feature extraction is shown in Fig. 3(c) for dataset 1 . The shape of a typical randomly selected putative ribosome extracted using semi-automated segmentation after denoising is depicted in Fig. 3(1), and is consistent with the expected shape of ribosomes based on previous electron microscopic studies.

Automated segmentation procedure: The next goal is to obtain automatic putative ribosome segmentation, for which we use template matching. In this technique, different parts of the volume are compared (e.g., cross-correlated) with the given template, which is an assumed model of the desired feature to 
locate places where the pattern of interest might be present. Our template-matching method focuses on exploiting some prior knowledge about the studied data; since the cytoplasm of the cell is always considered as the background, we find its average intensity by selecting a few sample points, and then fix it to zero. As a result of this fixed background, contrary to normalized cross-correlation, we do not shift the values of the volume during the matching process and thus we only have one scaling parameter. In addition, we enforce a minimum average intensity constraint on the ribosomes, which together with the previous condition decrease the number of false detections.

Since we are not provided with a template, we assume the ribosomes to be uniform spheres of a specified radius. Although this might not be the best approximation, this symmetry selection also eliminates the angular alignment which in turn reduces the problem to a much simpler one. In our approach, we find the values which minimize the following,

$$
\min _{\alpha, t} \int_{C}(\alpha(t) g(x)-f(x-t))^{2} d x=\min _{\alpha, t} \int(\alpha(t) g(x)-f(x-t))^{2} h(x) d x
$$

where $g(x)$ is the template (uniform sphere in our case), $f(x)$ is the given reconstructed tomogram, region $C$ is a sphere slightly bigger than the template where we compare the template with the sub-volume, $h(x)$ is a mask for that region; $h(x)=\left\{\begin{array}{ll}1 & x \in C \\ 0 & x \notin C\end{array}\right.$, $t$ is the translation parameter and $\alpha$ is a coefficient that might vary for each $t$ and allows the data to have a scaled version of the template. We also specify a threshold $\hat{\alpha}$ which is the minimum acceptable scaling coefficient. The above function is convex with respect to $\alpha$ and hence there is only one minimum which can be found by equating the derivative to zero,

$$
2 \int g(x)(\alpha(t) g(x)-f(x-t)) h(x) d x=0 .
$$

After the change of variable $y=x-t$ and using the fact that $g(x)$ is an even function (we consider a radially symmetric template), we have

$$
\alpha(t)=\frac{\int g(x) f(x-t) h(x) d x}{\int g^{2}(x) h(x) d x}=\frac{\int g(-y-t) f(y) d y}{\int g^{2}(x) d x}=\frac{1}{n} v(t)
$$

where $n=\int g^{2}(x) d x$ and $v(t)=(f * g)(-t)$. Note that we can always write the product $g(x) h(x)$ as $g(x)$, because the nonzero part of $g$ is included in the non-zero part of the mask. Therefore the problem is reduced to

$$
\min _{t} \int\left(\frac{1}{n} v(t) g(x)-f(x-t)\right)^{2} h(x) d x
$$

The minimization leads to

$$
\min _{t} D(t)=\min _{t}\left(c(t)-\frac{1}{n} v^{2}(t)\right) ; \alpha(t)=\frac{1}{n} v(t) \geq \hat{\alpha}
$$

where $c(t)=\left(f^{2} * h\right)(-t) \cdot D(t)$ is the least-squared error between the scaled template and the reconstructed tomogram. The computational complexity of the feature extraction algorithm is of the $O(N \log N)$, where $N$ is the number of voxels.

A test example is shown in Fig. 3(a)\&(b) for obtaining the centers of the ribosomes. We tried the feature extraction algorithm on non-denoised volumes and found that the algorithm did not pick any putative ribosomes. The putative ribosomes are extracted based on intensity, $\alpha(t)$ which needs to be greater than a specified threshold $\hat{\alpha}$, and also the least-squared error between the scaled template and the reconstructed tomogram, $D(t)$ (Fig. 3(k) for dataset 1). The original scale of the volume was $0.55 \mathrm{~nm} /$ pixel. Since the datasets were binned by a factor of 4 the scale is 2.2 $\mathrm{nm} /$ pixel accordingly. The size of each ribosome is about $20 \mathrm{~nm}$ across. The extracted putative ribosomes from the tomogram by template matching are sorted based on the error between the scaled template and the ribosomes in the tomogram to obtain the best matches. As the template, we used a uniform sphere of radius 4 pixels; this radius minimized the false positives and provided the best matches when compared against the semi-automated segmentation results (the true radius is $\sim 4.5$ pixels). Using the automatic procedure, we obtained 370 and 419 ribosomes for datasets 1 and 2 respectively. There were about 11 and 4 outliers using the automated technique for datasets $1 \& 2$ respectively. The semi-automated segmentation procedure obtained 363 putative ribosomes for dataset 1 and the comparison with the automated method is shown in Fig. 3(c)\&(d). The coordinates obtained using semi-automated and automated techniques were compared and about $85 \%$ of the ribsomes were within 5 pixels which is about half the ribosome size as shown in Fig. 3(i). About $92 \%$ of the ribosomes are within 7 pixels and the maximum error was within 10 pixels. The automatic segmentation for dataset 2 is given in Fig. $3(\mathrm{~g})$. From the quantitative analysis, the automated segmentation technique provides accurate estimates of the coordinates of the ribsomes compared to the semi-automated segmentation. Quantitative analysis such as ribosome density is shown in Fig. 3(h) and Table 1 and the distance to the nearest neighbor distribution is depicted in Fig. 3(j) and Table 1. From the ribosome density in Fig. 3(h) the ribosome count translates to 373.75 and 432 ribosomes for dataset 1 and 2 (not shown in the figure) respectively which is close to the results obtained using automatic procedure without the outliers (rows 4 and 5 of Table 1 ).

\begin{tabular}{c|c|c}
\hline & Dataset 1 & Dataset 2 \\
\hline Nearest Neighbor & Mean (pixels)-10.3 & 13.3376 \\
distribution & Variance (pixels)- 2.89 & 5.773 \\
Ribosome Distribution & $\mathrm{Avg} / \mathrm{nm}^{3}=2.10 \mathrm{e}-5$ & $1.81 \mathrm{e}-5$ \\
Average ribosome count & 373.75 & 432 \\
Automatic procedure & 359 & 415 \\
\hline
\end{tabular}

\section{Table 1. Quantitative analysis \\ 4. CONCLUSION AND FUTURE WORK}

Electron tomograms are intrinsically noisy and this poses significant challenges for image interpretation, especially in the context of low dose and high-throughput data analysis. Our goal has been to evaluate the relative performance of different denoising methods in further improving the SNR, and to test whether these denoised tomograms can be processed automatically to extract biologically relevant information. We show here that denoising significantly improves the fidelity of automated feature extraction. The NAD algorithm performs best for recovering structural information as the identification of putative ribosomes on denoised volumes using automated procedures closely matches those identified by user interaction (Fig. 3(i) and Table 1). We show that spatial information such as ribosome distribution can be obtained automatically from denoised tomograms and that the results closely match those obtained using semi-automated approaches. The use of these valuable computational tools provides a further step for quantitative analysis of 3D structures determined using electron tomography. As a part of future work, we intend to investigate the implications of electron doses and noise on denoising of tomograms and feature identification.

\section{REFERENCES}

[1] S. Subramaniam and J. L. S. Milne, "Three-dimensional electron microscopy at molecular resolution," Annual Review of Biophysics and Biomolecular Structure, pp. 141-155, 2004. 

structural information from cryo-electron tomograms," Current Opinion in Structural Biology, vol. 14, pp. 325-331, 2004. anisotropic diffusion," IEEE Transactions on Pattern Analysis and Machine Intelligence, vol. 12, pp. 629--639, 1990.

[4] G. Gilboa, N. Sochen, and Y. Y. Zeevi, "Image enhancement and denoising by complex diffusion processes," IEEE Transactions on Pattern Analysis and Machine Intelligence (PAMI), vol. 26, pp. 1020-36, 2004.

P. Kovesi, "Phase preserving denoising of images," The Australian Pattern Recognition Society Conference: DICTA'99, pp. 212-217, December 1999. Perth WA.

D. L. Donoho, "Denoising via soft-thresholding," IEEE

Transactions on Information Theory, vol. 41, pp. 613-627, May 1995.

J. Portilla, V. Strela, M. Wainwright, and E. P. Simoncelli, "Image denoising using scale mixtures of Gaussians in the wavelet domain," IEEE Transactions on Image Processing, vol. 12, pp. 1338-1351, November 2003.

[8] J. R. Kremer, D. N. Mastronarde, and J. R. McIntosh, "Computer visualization of three-dimensional image data using IMOD," Journal of Structural Biology, pp. 71-76, 1996.

[9] A. S. Frangakis and R. Hegerl, "Noise reduction in electron tomographic reconstructions using nonlinear anisotropic diffusion," Journal of Structural Biology, vol. 135, pp. 239250,2001

[10] J. L. T. Thong, K. S. Sim, and J. C. H. Phang, "Single-image Signal-to-Noise Ratio estimation," Scanning, vol. 23, pp. 328336, 2001 .

[11] J. Frank, Three-Dimensional electron microscopy of macromolecular assemblies: visualization of biological molecules in their native state: Oxford University Press, USA, 2006.

[12] "http://www.tgs.com/."

[13] J. Weickert, "Anisotropic diffusion in image processing," 1996.
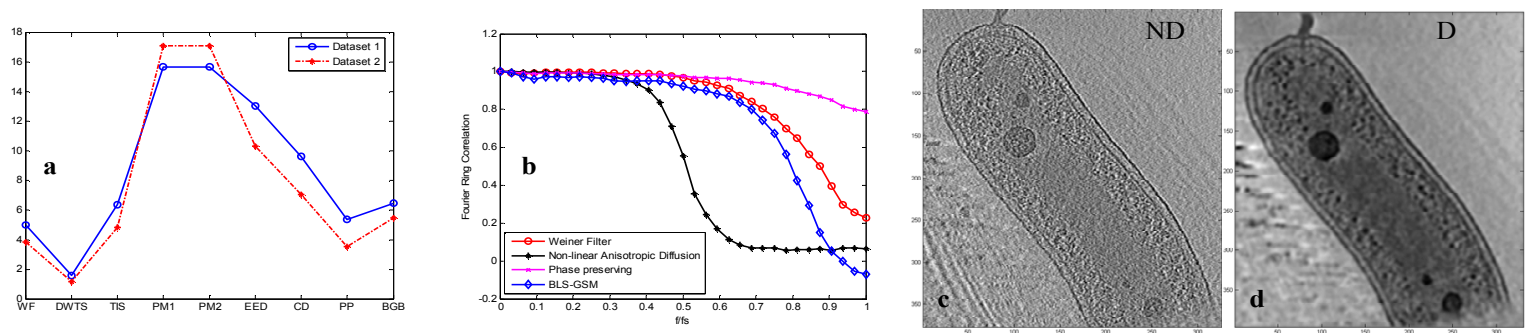

Fig. 2. (a) Single-image SNR for various denoising methods for cryo tomograms, (WF-Wiener Filter, DWTS- Discrete Wavelet transform with soft thresholding, TIS-Translation invariant with soft thresholding, PM1 and PM2-Perona-Malik equations, EED-Edge enhancing Diffusion [13], CD-

Complex Diffusion, PP-Phase preserving and BGB- BLS-GSM method. (b) Fourier Ring Correlation (FRC) for reconstructed Bdellovibrio tomograms using various denoising methods. Denoising of reconstructed Bdellovibrio tomogram (dataset 1$)$ using NAD (c) noisy \& (d) denoised.
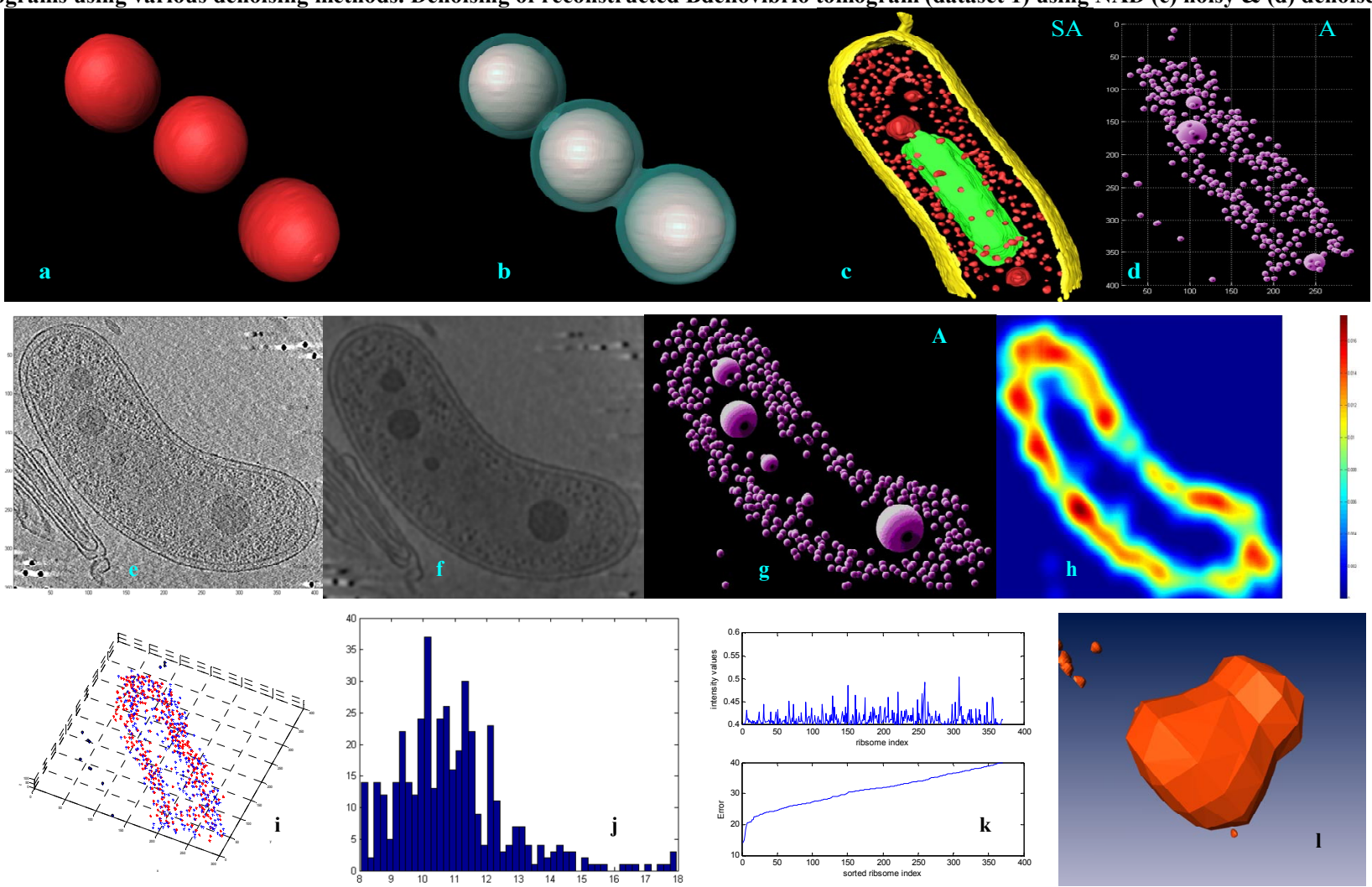

Fig. 3. Top Row (a-b): Test example to extract the ribosome, (c-d) Semi-automated and automated segmentation on dataset 1. Second Row (e-f): Comparison of the denoising results on dataset 2 using NAD. (g) Automated segmentation and (h) Ribosome density on dataset 2. Last Row (i):

Comparison of the locations of the extracted ribosomes using semi-automated (*) and automated segmentation ( + ), (j) Distance to the nearest neighbor distribution, (k) Intensity and error values for the extracted ribosomes for dataset 1 and (l) Shape of a random ribosome extracted after denoising. 